Cronfa - Swansea University Open Access Repository

This is an author produced version of a paper published in :

IEEE Computer

Cronfa URL for this paper:

http://cronfa.swan.ac.uk/Record/cronfa30966

\title{
Paper:
}

Jones, M. (in press). The Challenge and Opportunties of Interdevice Media. IEEE Computer

This article is brought to you by Swansea University. Any person downloading material is agreeing to abide by the terms of the repository licence. Authors are personally responsible for adhering to publisher restrictions or conditions. When uploading content they are required to comply with their publisher agreement and the SHERPA RoMEO database to judge whether or not it is copyright safe to add this version of the paper to this repository. http://www.swansea.ac.uk/iss/researchsupport/cronfa-support/ 


\title{
The Challenges and Opportunities of Inter-device Media
}

\author{
Timothy Neate ${ }^{\dagger}$, Matt Jones ${ }^{\dagger}$, Michael Evans ${ }^{\ddagger}$ \\ ${ }^{\dagger}$ FIT Lab, Department of Computer Science, Swansea University, Swansea, UK \\ ${ }_{\ddagger}^{\ddagger}$ BBC Research and Development, Salford, UK
}

\begin{abstract}
We live in a many-device, media-rich, connected world. But despite the great diversity of screens we regularly engage with, one still plays a prominent part in our homes - the television. While, previously, we might have browsed a paper magazine while halfwatching a TV programme, today we are more likely to be interacting with handheld devices. In the pre-digital era, choreographing and combining TV content with the viewers' other media was virtually impossible. Now, broadcasters and independent application designers are doing so, and are seeking ways to ensure that experiences that span devices are engaging, effective, and congruent. This article addresses the critical issue of supporting users in managing their attention across devices; uncovering the key attributes of experiences in which two or more devices are complementary.
\end{abstract}

\section{Second Screening}

A significant proportion of television viewing is accompanied by mobile device usage. In fact, this is quickly becoming one of the most ubiquitous multi-device use cases. A recent (2015) report suggests that $87 \%$ of us regularly engage with second screens while watching television [1]. This statistic appears to show growth since Google's 2012 consumer study [2], which found this figure to be $77 \%$ and that this was predominantly smartphones (49\%) and laptops/PCs (34\%). This strong increase of people interacting with devices while watching TV positions it as a potential exemplar of multi-device interaction and a clear area for pushing the frontiers of design.

Since the middle of the 21st century, the television has become a focal point of the modern home - often serving as a centrepiece, and a shared focus - a place for families to gather around to unwind and reflect on their day. Even now, in the smartest of 
homes its fundamental component (a large shared display for video consumption) normally remains - albeit in a more internet-enabled way. The large uptake of mobile devices in the living room has seen a large diversity of second screen interactions, which are mixed in terms of how related they are to the programme at hand. Rooksby et al. [3] and D'heer et al [4], when studying how people use their mobile devices while watching television, found that their usage is highly complex and nuanced - sometimes totally unrelated to the programme; sometimes totally related; often a mixture of both. The mobile device is highly personal and requires highly focused attention - Holz et al. [5] when studying how users use their second devices while watching television found that the device often acts as the primary screen, and the users tend to focus on this - switching back to the television when it piques their attention.

Though in many cases the digital worlds are disparate and unrelated, and users engage with material totally unrelated to the programme, much content browsing is also related to the television. A 2012 Google report suggests that $22 \%$ of simultaneous use is complementary - typically with a tablet $(40 \%)$ or a smartphone $(38 \%)$ [2]. Such interactions have their origins in the early days of television - we have always wanted to get involved with the events on screen. Take for example, a 1970s British TV programme - The Golden Shot, in which a member of the audience (at home) would direct a blindfolded person to shoot a target over the phone while watching television [6] - such interactions are now reflected in playalong games. And viewer-television interactions such as phone-ins and letters have now been pushed into the digital age through social media - e.g. people tweeting along with live debate (c.f. [7]). Now, in more recent times, this explosion of internet-equipped, interactive, powerful devices into our living rooms has borne new exiting ways for content creators to support these behaviours in real time.

\section{Companion Applications}

Many popular programmes now have an associated second screen app, which is designed to run alongside the programme, allowing for real time feedback and interaction with a 'live' programme. Popular examples of this include interactive apps for talent shows - the Britain's got Talent ${ }^{1}$, and the X Factor ${ }^{2}$ apps, for example, allow users to play along with online events - vote off acts, and join in polls. There are also applications which provide trivia about a given programme, such as the Breaking Bad app ${ }^{3}$.

Users with multi-platform ecologies such as Amazon Fire TV, and Google Chromecast, are now becoming familiarised into many-screen media consumption. Many application developers and broadcasters have began converging on the creation of multi-

\footnotetext{
${ }^{1}$ Britain's Got Talent app: https : / /goo.gl/7Yx09W

${ }^{2}$ X-factor App: https: / / goo.gl/g JRhNa

${ }^{3}$ Breaking Bad App: http://goo.gl/3MwMKp
} 
device, non-linear, television experiences. The second screen use case has led to investigations from broadcasters into how they can support such second screen behaviours. They wish to close the loop for their consumers and make the second screen experience more focused, and with a lower barrier to entry. Therefore, a plethora of second screen apps for television has come about to fulfil a variety of purposes - interactive quiz applications ${ }^{4}$, social media extensions of a programme [8], and supplementary material such as additional facts or complementary audio/visual content [9]. The BBC term such second screen materials as 'Companion' Applications. Such second screen apps are driven by our need to search for more; to enquire in the attention lulls of in a programme and to focus on the programme in the peaks.

Many extensive deployments of companion applications have aimed to push forward the boundaries such applications, for example: Murray et al's Story-Map application to help users conceptualise long form narratives [10]; and Dowell et al.'s companion application which aimed to support users in understanding complicated concepts in a programme about physics through interactive graphs. In essence, through a variety of methods, broadcasters and application developers are now explicitly encouraging users to engage with related, time-relevant, second screen material. And due to the limits of human attention this, implicitly, suggests that the users will have to actively ignore some content on the 'other' display to engage properly with material on the attended display.

The remainder of this article presents our research on designed multi-screen experiences, and looks at how our research, along with that of the HCI (Human-computer Interaction) community, has endeavoured to understand, overcome, and ultimately improve design in this area. We explore the results of an online survey and a series of interviews we undertook to better understand the requirements of users when they actively search on a second screen from the perspective of attention requirements, and then go on to discuss some of our interventions towards alleviating these issues.

\section{The Bottlenecks of Multi-device Media}

Television, as a media, is relatively passive - compared to, say, reading. To read, we must investigate line by line, interpret the semantic information, conceptualise its meaning, and attach this to an audio-visual narrative. This requires a degree of mental effort. With television, however, the interpretation of the script and intent of the author is sometimes explicitly laid bare for us - resulting in lower levels of effort to engage. Research by Schmitt et al. in 2003 [12] suggests that the attention-low nature of some programming generally supports side activities such as social interaction, reading, or eating. In fact, their study indicated that even in 2003 (pre-digital ubiquity)

\footnotetext{
${ }^{4}$ Antiques Roadshow Playalong App: http: / / goo.gl/RI IpFu
} 
we were engaged with side activities $46 \%$ of the time we were watching television. This being said, though television is a relatively un-taxing media, adding an additional activity creates a divided attention scenario - often meaning the television fades into the background. The addition of an interactive information rich second screen, with an increased number of user behaviours, exacerbates this side activity - meaning that we can become totally lost in our devices - ignoring the television.

From an ergonomic view, second screening typically involves us resting the device in our laps, or cradled in our hand - but generally in the periphery of our vision (see Figure 1). Brown et al. [13] phrase this as the 'sit back' nature of the scenario. This hinders our ability to shift attendance to the secondary screen or to the TV, as we are required to significantly adjust our gaze and/or posture, adding increased switching cost.

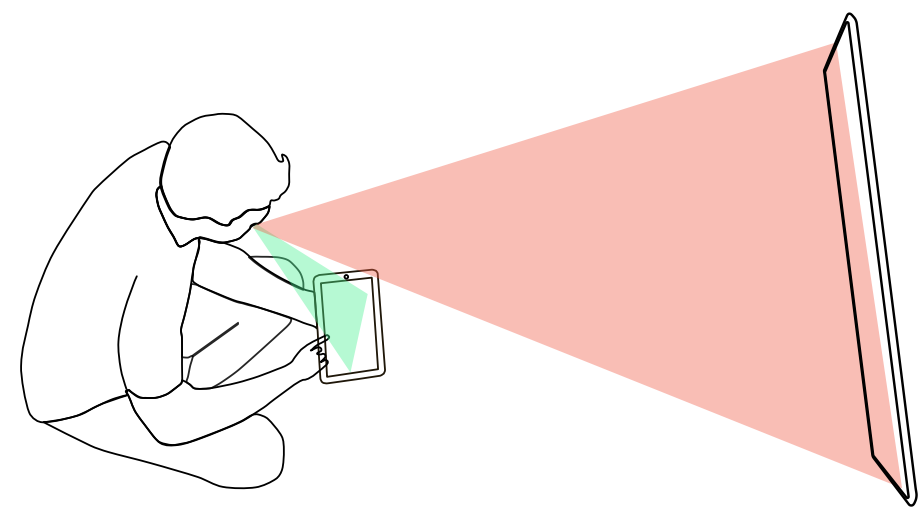

Figure 1: To engage with both devices, a user must switch their gaze significantly. The unattended device is visible only in the corner of their eye. For example, to switch engagement from the tablet (green) to the TV (red) the user has to physically alter their position.

The work on attention factors in multi-screen systems is rich and growing year on year in the human-computer interaction community, but does not encompass multi-screen media, as this is a relatively new concept. Recent lab studies by Brown et al. [13] and Holmes et al. [14] document device interplay between the a tablet the television through eye-tracking. As the device is in the peripheral of our vision it means that users must manage their own gaze, which adds additional attention requirements detracting from the experience. In addition, psychological factors such as our poor simultaneous comprehension of text and auditory information is also likely to limit our potential engagement with such experiences.

Further to the physical discontinuity between the devices, a notion of 'information overload' is a recurring theme in the literature on companion applications. For exam- 
ple, Geerts et al. [15] who, when trialling a dual-screen application for a programme noted that, the users were often overcome with the stimuli, and found that they had to return later when the pace of the TV programme had slowed down. A problem when returning later, however, is the fact that the information may no longer be relevant to the TV programme, and may only act as a recap. Such experiences are often viewed as cognitively involving, and not seen as something someone may 'unwind' to [16]. The drivers of attention overload in this scenario, however, have not been investigated thoroughly.

\section{The Viewers' Perspective}

No work currently looks at the issues around second screen attention when browsing second screen content, from the perspective of the users, and their regular viewing habits. If we are to consider the design of applications which support the complementary browsing of second screen materials, it is essential that we understand this phenomenon from the viewers' detailed perspective. Therefore, we conducted two studies to investigate this from the user's perspective - through an online survey and in-depth interviews with second screeners, we looked to uncover reflective insight from a large population.

\section{Online Survey}

As a starting point, we surveyed people who regularly watched TV and used a second screen at the same time to engage with complementary material. With regards to our participant pool, we recruited from university mailing lists at Swansea University (UK), seeking people who actively browse complementry content or social media while watching television - we sent out an request to university mailing lists - both staff and students - receiving 364 responses in total. Though we explicitly noted in the email we were interested in those who browsed complementary materials, we filtered out those who did not with the qualifying question "Do you ever engage with additional content related to the show you are watching on a secondary device, such as a tablet computer or smartphone?" - reducing the total number of participants for our analysis to 260 . Of our participants, the average age was $21.4(\max =42, \min =$ 18), of which 151 identified as male, 108 female and 1 gender fluid.

As the participants were from a UK university they were from a number of countries and spoke a variety of languages, but were predominantly English-speaking British nationals. In addition, our participants were frequent touch screen users - 79\% (205) participants 'strongly agreed' that they used touch screens often. Moreover, a large number of people also sided towards agreement with the fact that they are easily distracted by their mobile devices 85 strongly agreed, 61 agreed. We also asked the types of second screen viewing they undertook - the key trends of our participants were that they: searched for content related to the production of the programme (104) - 
actors, directors, plot lines, etc; facts related to the programme (53); and social network related browsing (20). The participants showed a strong preference for mobile devices such as smartphones (138 participants), but also used tablets (28 participants) and laptops (36 participants).

We were interested in finding out how second screening on a mobile or tablet while watching had an impact on their experience of one or both of their devices. Through the online survey we asked participants specific questions to whittle down the issues when they second screened. We asked questions associated to their mental effort; questions that probed how certain types of programming or applications affected their dual-screening; and to what extent different stimuli types (auditory, visual) interact and affect the users. The questions, and respective results are depicted in Table 1. For questions 8 and 11 we left free text fields and asked participants to provide explicit examples when necessary.

\begin{tabular}{|c|c|c|c|c|c|c|c|}
\hline Statement & Measure (1 - 5) & 1 & 2 & 3 & 4 & 5 & Median \\
\hline I- When I watch TV I experience & Low/High Mental Effort & 50 & 89 & 90 & 23 & 8 & 2.0 \\
\hline 2 - When I watch TV with a secondary device I experience & Low/High Mental Effort & 26 & 92 & 88 & 46 & 8 & 3.0 \\
\hline 3 - When I watch TV and search for related information this requires & Low/High Mental Effort & 21 & 97 & 77 & 57 & 8 & 3.0 \\
\hline 4-When I watch TV while social networking this requires & Low/High Mental Effort & 36 & 92 & 75 & 47 & 10 & 3.0 \\
\hline 5 - While engaged with a second screen I am aware of visual content on the TV & Strongly Disagree/Agree & 9 & 51 & 69 & 102 & 29 & 4.0 \\
\hline 6 - While engaged with a second screen I am aware of auditory content on the TV & Strongly Disagree/Agree & 11 & 32 & 62 & 93 & 62 & 4.0 \\
\hline 7 - When I watch TV I am aware of visual information on my handheld device & Strongly Disagree/Agree & 8 & 40 & 52 & 126 & 34 & 4.0 \\
\hline 8 -Certain types of programme require more mental effort when second screening & Strongly Disagree/Agree & 0 & 12 & 17 & 92 & 139 & 5.0 \\
\hline 9-I am likely to focus on the second screen content more than the TV & Strongly Disagree/Agree & 15 & 59 & 85 & 80 & 21 & 3.0 \\
\hline 10 - I am likely to focus more on the TV programme when I second screen & Strongly Disagree/Agree & 13 & 74 & 96 & 54 & 23 & 3.0 \\
\hline 11 - I am more likely to use a second screen for some programmes than others. & Strongly Disagree/Agree & 19 & 25 & 34 & 104 & 78 & 4.0 \\
\hline
\end{tabular}

Table 1: Data from the online questionnaire: the numbers reported refer to the number of participants $(N=260)$ who responded with the stated answer, along with the median of all responses. Questions 1 to 4 relate to the users' perceived level of low/high mental effort $($ Low Mental Effort = 1, High Mental Effort = 5), and questions 5 to 11 refer to their agreement with the statement $(1=$ Strongly Disagree, $5=$ Strongly Agree).

The quantitative data we received suggests that to engage with TV on its own requires little mental effort. But, with the introduction of a handheld device we found that the required increased this significantly. The additional effort reported by the participants to engage with complementary content as opposed to social networking may be explained by the fact that tangential search of information is an active search process. Such findings bolster the need for designated companion applications that support users when watching TV programmes - essentially reducing the need for users to formulate and pursue knowledge query. Participants generally reported noticing the auditory channel of the TV more - this is not surprising as audio is omnidirectional, and one must actively engage with the visual stimuli to view it. However, what this makes clear is that when second screening, users are always listening. As textual comprehension drops significantly when monitoring auditory information, this is likely a major impacting factor on the perceived additional attention cost of the dual-screen scenario. 
The qualitative data strongly suggests that certain types of programme impact strongly on the dual-screen experience. In terms of genre the participants tended to cite programming that required constant attention because it was fast changing or cognitively involving (documentaries), or if the experience was largely dependent on prior knowledge of prior/current developments (drama) - see Figure 2 for a full picture. Further, a clear trend was driven by the physiological factors around this - use cases that require frequent visual focus, like a character's nuanced expression in a drama, a complex diagram in a documentary, or indeed the presentation of subtitles, are problematic for this second screen use cases as they require users to systematically 'follow along' with the developments.

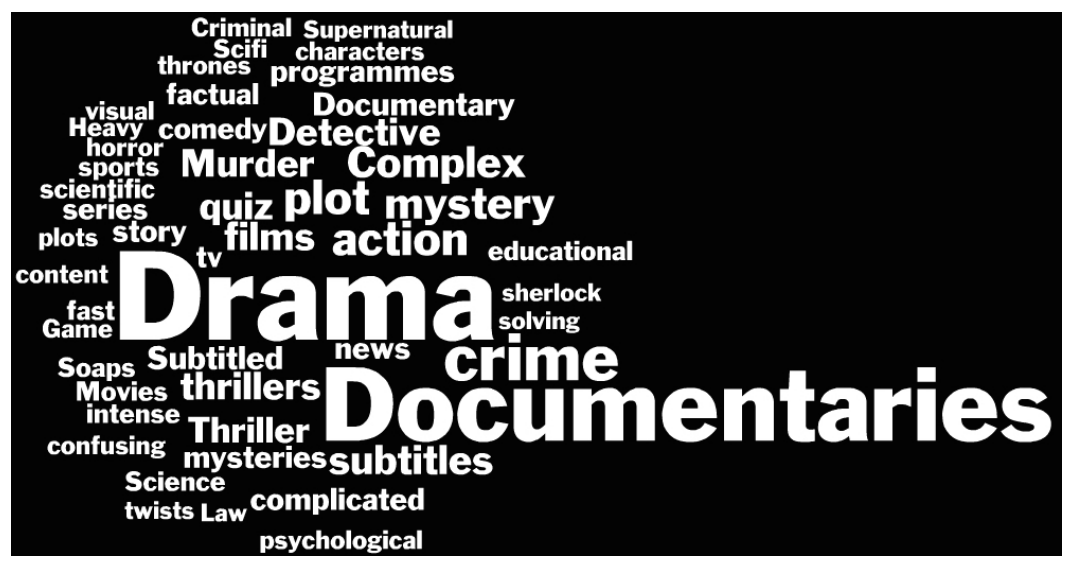

Figure 2: This wordcloud represents the $\mathbf{5 0}$ most frequently noted phrases that users stated when asked what types of programme require more mental effort for them to second screen alongside. The size of the words is scaled to their frequency - the most frequent being documentaries and drama and therefore the largest.

\section{Interviews}

With an overview of the types of programming that require extra effort on the user's behalf, we now discuss interviews which probe deeper to gain insight. We conducted semi-structured interviews with 20 participants to further understand what impacts our attention, along with how they manage their attention to avoid, and overcome the attention bottlenecks associated with this use case. As with the online questionnaires, we recruited participants who actively engaged in second screening that extended their television viewing to varying degrees - our participants were all members of the British public with an average age of 39 ( $\mathrm{SD}=13.04)$ - 11 males, 9 females, who, on average, watched 2 hours and 5 minutes of TV per day. The participants were all generally from a technologically aware demographic -16 people strongly agreed 
that they regularly engage with touch screens (tablets or smartphones) and 4 agreed. Also, many (16/20) of the participants noted that they were often engaged in other non-second screen activities as well as watching television- such as talking to their partner, or eating.

Due to the semi-structured nature of the interview, the test coordinator was free to probe deeper into certain points, and to be tangential in questioning. However, our fixed questions investigated: whether the participants felt 'information overload' at any point when second screening, and how they adapt their viewing; and to note points when participants would sacrifice not looking at their secondary device to look at the TV (and vice versa).

Two common themes emerged from our questioning: mitigating against overload, and compensating for overload:

Mitigating against overload $-74 \%$ of our participants noted they often get some degree of information overload when trying to divide their attention between screens. To mitigate against missing materials our participants commonly noted: actively putting aside the device in more engaging parts of the programme (6 participants), pausing to read/view something on a second device (6 participants), or trying to focus on one screen and dip in and out of the other dependent on what attracts their attention ( 9 participants). Of those who tried to dip in and out of content we noted two main viewing habits: if the show was something the participant wanted to focus on, they would view the TV as the primary device and dip out when their mobile caught their attention (e.g. a notification) (5 participants) - " If I am watching TV, I am watching TV. I tend not to get distracted and tend to fixate on one thing. I guess I go into some kind of bubble 'experience' about what is on the TV. I want a full on experience". Those who treated the the mobile device as the main screen tended to pick up on auditory cues - "if it's not a team I'm invested in, but a team I'm just watching because I like watching football then it will be the other way around. And I will just look up occasionally when I hear the crowd noises, or the commentator get excited." (P13)

Compensating for overload - To compensate for overload (i.e. the user has missed some information) some common trends emerged. The most frequent comments were related to rewinding the programme to catch up on key events (8 participants) they missed while interacting with their devices - "Yeah, well you can rewind it now, but it can take twice as long to watch a programme. Because obviously, you're not paying attention and you have to go back and check what you were watching" (P19) This appeared to be a majorly common occurrence, with more than $50 \%$ of the participants who dual screened undertaking this.

These results suggest that we should allow users to 'take a break' and catch up with television/companion material for when users miss content. In addition, those who 
wish to design companion applications should heed the warnings regarding the amount of times our participants were taken out of the television experience by their mobile devices. Simple interventions can be realized to overcome the more significant issues - for example by supporting common user behaviours such as pausing and rewinding content when they are mitigating or compensating for overload. The companion device should be aware of this, and behave appropriately - for example, remain within the same timeline as the programme by 'rewinding' and 'pausing' with it.

Though keeping the devices in synchronization when the TV programme is rewound/paused is a good method by which designers can allow for the mitigation and compensation of content lost, they are still retrospective solutions to the problem of users becoming overloaded by second screening. Our research looks to consider how we may design companion experiences so that users are not overcome by materials in the first place. We now discuss some of our interventions towards the design of cross-device media - we discuss a series of interventions we have implemented and studied empirically towards compensating for some of the issues evidenced in this section.

\section{Designing for Cross-device Media}

Previously, where we may have sat with a newspaper - our attention consumed in its pages - we may have ignored a large portion of the television (and vice versa). As the newspaper is static this was not of major concern. However, if we wish to provide updating, time-relevant information, or related social media - with the explicit intention of embellishing a television programme - such experiences should consider the finite cognitive resources of our users. Currently the dual-screen scenario is undesigned. To compensate for the complexities of time-relevant cross-device media, we should consider strongly the design of such experiences.

Human factors is a fundamental consideration in the adoption of a new technology the envisioned future of a connected multi-device home cannot prosper without its design considered throughly. To this end, much work has looked at how we may consider the design of cross-device media. Work has aimed to understand this scenario from a human factors perspective. The community has explored deployments of interactive cross-device experiences (c.f. [16]) and lab studies (c.f. [13, 14]) which have aimed to better understand the multi-device experience. Our work extends such research by considering interventions - ways by which cross-device media may be improved through altering its fundamental design. So far, in our work we have explored two main avenues - mediating attention, and designing visual complexity. 


\section{Mediating Attention}

Display switching costs are a major factor when users second screen - as noted in Figure 1, the disjunct between the device and the television often means that one must physically adjust their body, visual focus, and concentration to view content. Our work, published in CHI 2015, has investigated how we may compensate for this by directing, or mediating attention [17]. We explored how attention could be directed in a dual-screen scenario, and by doing so, aimed to solve two major issues - users missing content on a secondary device in their peripheral; and, users becoming conscious that they are missing content, therefore checking the device when nothing has updated.

We conducted a systematic study with 18 participants in which we introduced pieces of companion content on a second screen (a tablet computer) accompanied with a different style of notification. We looked at both auditory and visual notifications, both on the device and on the television. This enabled us to explore methods by which content creators can affect the way a user divides their attention between the screens at certain points in a multi-device media experience. We explored this by investigating the effect of auditory stimuli on a tablet, visual stimuli on the tablet, and visual notifications on the television. We noted that the peripheral stimuli yielded the fastest reaction times (especially auditory) and that feedback on the television actually resulted in slower reaction times than no stimuli.

We also looked at the subjective impact with our participants and found that auditory icons - sounds related to the show - could act as an effective 'anchor' - tying the television programme to the second screen companion content. In addition, the notifications on the television were also responded to positively - they often afforded the participants the ability to defer their attention, and look at the second screen content in their own time.

From this study we learned that users prefer their attention mediated by the experience itself - that, when directed, their experience of the content is enhanced. Our research outlined clear findings for content creators - that when time-relevant content is presented we can use peripheral on-device (especially auditory) methods to gain attention, and when the content is not time critical, a degree of user autonomy can be afforded through TV-based notification methods.

\section{Quantifying Dual-screen Visual Complexity}

Following up this work we wanted to understand some of the fundamental factors behind dual-screen complexity (and therefore attention overload) from an empirical perspective. In our work, published in CHI 2016 [18] we conducted an empirical study to investigate the factors which constitute complex television. To do so we pre- 
sented participants with second screen materials of differing complexity (see Figure 3 ), and measured the objective and subjective impact of how the material affected the dual screen experience.

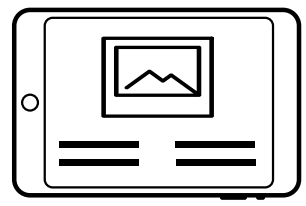

$\operatorname{tg}$

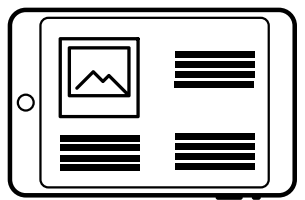

$\operatorname{Tg}$

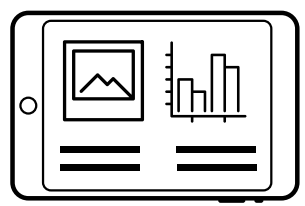

tG

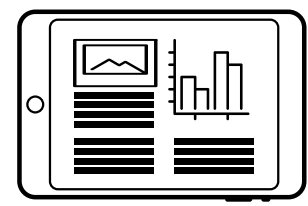

TG

Figure 3: From a survey of available companion applications, we derived some typical complexity levels (informed by reliable metrics such as the Flesch-Kincaid reading age [19]). We then created typical content at four varying complexity levels - 'tg' (simple text, simple graphics), 'Tg' (complex text, simple graphics), 'tG' (simple text, complex graphics), and 'TG' (complex text, complex graphics).

Our results indicated that textual complexity was the largest driving factor for the participants' attention. This resulted in significantly more visual focus on the device (in terms of time), and increased workload for the participants - they found it significantly more challenging to balance their attention across the screens when the textual complexity was higher. This was supported by the interview data, and the qualitative feedback from questionnaires.

From the semi-structured post-study interviews we were able to establish some thematic relationships between complexity on the television, and specific types of programming. We saw three common factors in the qualitative data for what constituted complex television content: Intense Dialogue - complex descriptions or multiple people speaking simultaneously; Novelty - when a new person appears, or there is a new scene; Semantically Vital Visual Information - essential information on the TV that we cannot infer from the auditory channel.

\section{Complexity Curation: Adapting UI around Complexity Heuristics}

Our previous experiment had determined some indicative factors for dual-screen complexity. The next step was to look at how we could use this information to adapt the complexity on a second screen device to balance for the perceived complexity on the television [18]. We explored two main concepts:

- Complexity Curation - adjusting the complexity on the second screen to account for the perceived complexity of the television content each time the par- 
ticipant moves to a new screen in the app. See Figure 4 for more detail.

- Complexity Adaptation - allowing the user to adapt the complexity themselves in a 'more/less info' interface.

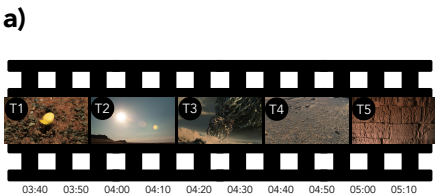

Time (mm:ss) b)

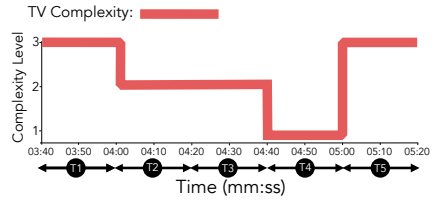

c)

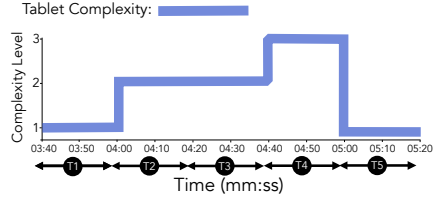

Figure 4: Complexity curation process: the programme is divided into time segments, from which a complexity level is determined. Then we inversely map this complexity onto the tablet. For example at 3:40 the complexity on the TV is considered HIGH complexity (3), therefore we render the complexity on the tablet as LOW complexity.

We conducted an experiment to determine if complexity curation is a viable method of compensating for dual-screen complexity. To do so we built a browsable second screen application that, when a user moves to a new page, would lookup to check the level of complexity to present. The textual information was then dynamically rendered at one of three complexity levels, dependent on the complexity of the TV content (as determined by our heuristics).

By comparing these to a baseline (random updating complexity) we were able to establish that curation was effective - despite the random and the curated case having the same averaged level of complexity, our research indicated that ramping up the complexity when TV complexity was likely to be perceived as low, and ramping it down when likely to be perceived as high worked as a method for balancing the complexity across the screens, which in turn enhanced the user experience. Further, we found that allowing the participants to adjust the complexity themselves, though useful, led to an increased interaction cost.

In general we suggest that the given complexity of a piece of companion material may be more taxing on the attention of our users than a designer may presume. Therefore, we suggest that designers consider strongly the complexity of the content that they introduce, and the impact it will have on the cognitive load of their users. Regardless of whether the complexity of the supplementary content is arrived at through artistic decision, trials with users, or is calculated algorithmically, for example through sensing their engagement through physiological data (see [20] for a discussion); the timing of complex content should be curated with the utmost importance when crafting multidevice experiences. 


\section{Conclusions}

Recent explorations around the area of multi-screen HCI for media contexts indicate great opportunities for the design of interactive experiences. And the large-scale adoption of mobile devices in our living rooms provides a great design lever by which content designers can create more engaging material for their users. These benefits, however, are not without their challenges. In terms of human factors, it is clear that introducing content additional to the television, which the user is encouraged to engage with, can create great perceptual dissonance. For example, in this paper, we detailed extensive issues users face when browsing content on a second screen, to the extent where they are regularly missing content - a lot of which is retrospectively compensated for (e.g. rewinding), or mitigated against (e.g. pausing). We believe that such retrospective solutions to missing materials on either the television or a mobile device are clumsy and will affect user experience negatively. Therefore, in this paper, we utilise the findings of several empirical studies to consider further how we can improve inter-device experiences in terms of attention management.

In sum, we believe that the successful adoption of such technologies in the future will not only pivot around hardware and software capabilities, but also around how readily such technologies are adopted. By designing technologies that support positive user experience through informed design, we see a promising future for inter-device media media. We envision cross-platform experiences wherein the content creator takes on additional role as a conductor of inter-device attention, towards providing immersive and, ultimately, designed multi-device experiences.

\section{References}

[1] G. Mann, F. Venturini, R. Murdoch, B. Mishra, G. Moorby, and B. Carlier, "Digital video and the connected consumer," Accenture, Tech. Rep., March 2015.

[2] Google, "The new multi-screen world:understanding cross-platform consumer behaviour." Google, 2012. [Online]. Available: http://goo.gl/xdbOe1

[3] J. Rooksby, M. Rost, A. Morrison, M. Bell, M. Chalmers, and T. Smith, "Practices of parallel media: Using mobile devices when watching television," in CSCW - Designing with Users for Domestic Environments: Methods, Challenges and Lessons Learned. ACM, Feb. 2014.

[4] E. D'heer, C. Courtois, and S. Paulussen, "Everyday life in (front of) the screen: The consumption of multiple screen technologies in the living room context," in Proceedings of the 10th European Conference on Interactive Tv and Video, 
ser. EuroiTV '12. New York, NY, USA: ACM, 2012, pp. 195-198. [Online]. Available: http://doi.acm.org/10.1145/2325616.2325654

[5] C. Holz, F. Bentley, K. Church, and M. Patel, "'i'm just on my phone and they're watching tv": Quantifying mobile device use while watching television," in Proceedings of the ACM International Conference on Interactive Experiences for TV and Online Video, ser. TVX '15. New York, NY, USA: ACM, 2015, pp. 93-102. [Online]. Available: http://doi.acm.org/10.1145/2745197.2745210

[6] ATV. The golden shot (associated television). [Online]. Available: https: //www.youtube.com/watch?v=8grjSpkm_r4

[7] M. T. Maruyama, S. P. Robertson, S. K. Douglas, B. C. Semaan, and H. A. Faucett, "Hybrid media consumption: How tweeting during a televised political debate influences the vote decision," in Proceedings of the 17th ACM Conference on Computer Supported Cooperative Work \&\#38; Social Computing, ser. CSCW '14. New York, NY, USA: ACM, 2014, pp. 1422-1432. [Online]. Available: http://doi.acm.org/10.1145/2531602.2531719

[8] K. Kusumoto, T. Kinnunen, J. Kätsyri, H. Lindroos, and P. Oittinen, "Media experience of complementary information and tweets on a second screen," in Proceedings of the 22Nd ACM International Conference on Multimedia, ser. MM '14. New York, NY, USA: ACM, 2014, pp. 437-446. [Online]. Available: http://doi.acm.org/10.1145/2647868.2654925

[9] J. Dowell, S. Malacria, H. Kim, and E. Anstead, "Companion apps for information-rich television programmes: representation and interaction," Personal and Ubiquitous Computing, p. 14, 2015. [Online]. Available: https://hal.inria.fr/hal-01174692

[10] J. Murray, S. Goldenberg, K. Agarwal, T. Chakravorty, J. Cutrell, A. DorisDown, and H. Kothandaraman, "Story-map: Ipad companion for long form tv narratives," in Proceedings of the 10th European Conference on Interactive Tv and Video, ser. EuroiTV '12. New York, NY, USA: ACM, 2012, pp. 223-226. [Online]. Available: http://doi.acm.org/10.1145/2325616.2325659

[11] J. Kramskoy, "Orchestrated media - beyond second and third screen," February 2011. [Online]. Available: http://www.bbc.co.uk/rd/blog/ 2011-02-orchestrated-media-beyond-se-1

[12] K. L. Schmitt, K. D. Woolf, and D. R. Anderson, "Viewing the viewers: Viewing behaviors by children and adults during television programs and commercials," Journal of Communication, vol. 53, no. 2, pp. 265-281, 2003. [Online]. Available: http://dx.doi.org/10.1111/j.1460-2466.2003.tb02590.x

[13] A. Brown, M. Evans, C. Jay, M. Glancy, R. Jones, and S. Harper, "Hci over multiple screens," in CHI '14 Extended Abstracts on Human Factors in Computing Systems, ser. CHI EA '14. New York, NY, USA: ACM, 2014, pp. 665-674. [Online]. Available: http://doi.acm.org/10.1145/2559206.2578869 
[14] M. E. Holmes, S. Josephson, and R. E. Carney, "Visual attention to television programs with a second-screen application," in Proceedings of the Symposium on Eye Tracking Research and Applications, ser. ETRA '12. New York, NY, USA: ACM, 2012, pp. 397-400. [Online]. Available: http://doi.acm.org/10.1145/2168556.2168646

[15] D. Geerts, R. Leenheer, D. De Grooff, J. Negenman, and S. Heijstraten, "In front of and behind the second screen: Viewer and producer perspectives on a companion app," in Proceedings of the 2014 ACM International Conference on Interactive Experiences for TV and Online Video, ser. TVX '14. New York, NY, USA: ACM, 2014, pp. 95-102. [Online]. Available: http://doi.acm.org/10.1145/2602299.2602312

[16] S. Basapur, G. Harboe, H. Mandalia, A. Novak, V. Vuong, and C. Metcalf, "Field trial of a dual device user experience for itv," in Proceedings of the 9th International Interactive Conference on Interactive Television, ser. EuroITV '11. New York, NY, USA: ACM, 2011, pp. 127-136. [Online]. Available: http://doi.acm.org/10.1145/2000119.2000145

[17] T. Neate, M. Jones, and M. Evans, "Mediating attention for second screen companion content," in Proceedings of the 33rd Annual ACM Conference on Human Factors in Computing Systems, ser. CHI '15. New York, NY, USA: ACM, 2015, pp. 3103-3106. [Online]. Available: http://doi.acm.org/10.1145/2702123.2702278

[18] T. Neate, M. Evans, and M. Jones, "Designing visual complexity for dual-screen media," in Proceedings of the 2016 CHI Conference on Human Factors in Computing Systems, ser. CHI '16. New York, NY, USA: ACM, 2016, pp. 475-486. [Online]. Available: http://doi.acm.org/10.1145/2858036.2858112

[19] P. Kincaid, R. Fishburne, R. Rogers, and B. Chissom, "Derivation of new readability formulas (automated readability index, fog count and flesch reading ease formula) for navy enlisted personnel," United States Naval Education and Training Support Command, Tech. Rep. 8-75, February 1975.

[20] C. Jay, S. Harper, A. Brown, M. Glancy, and M. Armstrong, "Attention approximation: from the web to multi-screen television." TVUX-2013, 2013. 